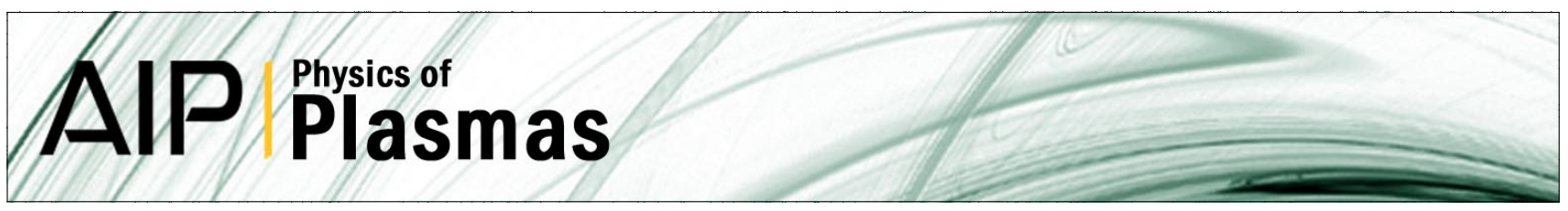

\title{
Properties of the Lenard-Balescu collision operator: A numerical study
}

Paolo Ricci and Giovanni Lapenta

Citation: Phys. Plasmas 9, 430 (2002); doi: 10.1063/1.1418720

View online: http://dx.doi.org/10.1063/1.1418720

View Table of Contents: http://pop.aip.org/resource/1/PHPAEN/v9/i2

Published by the American Institute of Physics.

\section{Related Articles}

Average atom transport properties for pure and mixed species in the hot and warm dense matter regimes Phys. Plasmas 19, 102709 (2012)

Hot-electron generation by "cavitating" Langmuir turbulence in the nonlinear stage of the two-plasmon-decay instability

Phys. Plasmas 19, 102708 (2012)

Spherical torus equilibria reconstructed by a two-fluid, low-collisionality model

Phys. Plasmas 19, 102512 (2012)

Space-charge-based electrostatic plasma confinement involving relaxed plasma species

Phys. Plasmas 19, 102510 (2012)

Numerical study of the characteristics of the ion and fast atom beams in an end-Hall ion source

J. Appl. Phys. 112, 083301 (2012)

\section{Additional information on Phys. Plasmas}

Journal Homepage: http://pop.aip.org/

Journal Information: http://pop.aip.org/about/about_the_journal

Top downloads: http://pop.aip.org/features/most_downloaded

Information for Authors: http://pop.aip.org/authors

\section{ADVERTISEMENT}

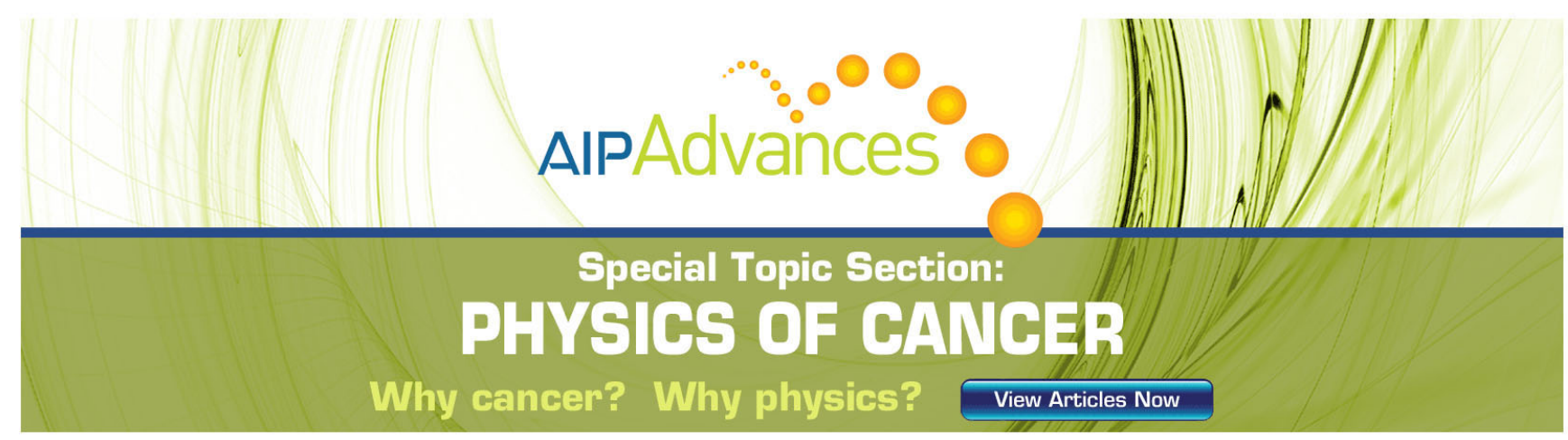




\title{
Properties of the Lenard-Balescu collision operator: A numerical study
}

\author{
Paolo Ricci \\ Istituto Nazionale per la Fisica della Materia (INFM) and Dipartimento di Energetica, Politecnico di \\ Torino, Corso Duca degli Abruzzi 24-10129 Torino, Italy \\ Giovanni Lapenta ${ }^{\text {a) }}$ \\ Istituto Nazionale per la Fisica della Materia (INFM), Unitá del Politecnico di Torino, Italy \\ and Theoretical Division, Los Alamos National Laboratory, MS:K717, Los Alamos, New Mexico 87545
}

(Received 27 July 2001; accepted 25 September 2001)

The kinetic evolution of a one-dimensional electrostatic plasma is discussed, comparing the different predictions of three kinetic models: the Vlasov model, the Lenard-Balescu model, and the exact transport model. The attention is focused on the Lenard-Balescu approach whose properties are studied in detail. The kinetic parameters present in the Lenard-Balescu collision operator are studied analytically and the kinetic equation with the Lenard-Balescu collision operator is solved numerically. The numerical solutions are compared with the results of an exact microscopic model and found in remarkable agreement. (C) 2002 American Institute of Physics.

[DOI: $10.1063 / 1.1418720]$

\section{INTRODUCTION}

In the present paper, we investigate the properties of the Lenard-Balescu collision operator. ${ }^{1,2}$ We will attack the problem using primarily numerical methods; while an extensive literature is available on theoretical studies of the Lenard-Balescu equation, ${ }^{1-4}$ its numerical solution is largely not explored.

Our study uses a relatively simple, but still meaningful, mathematical model of a one-dimensional electrostatic plasma. We consider a plasma model similar to the one studied in a series of previous studies (e.g., Dawson ${ }^{5,6}$ and Rouet and Feix ${ }^{7}$ ). The advantage of this choice is the availability of previous results to validate the numerical integration of the Lenard-Balescu equation, as it is possible to simulate the evolution of this plasma with an exact microscopic algorithm.

The plasma considered consists of a large number of electrons, interacting via electrostatic forces. The electrons are immersed in a uniform fixed neutralizing background. This background represents the ions which, being much heavier than the electrons, can be considered motionless for the time scale considered here. Initially, the electrons are distributed uniformly in space and their velocity distribution is not in thermodynamic equilibrium (i.e., it is nonMaxwellian). It has been pointed out by Rouet and Feix ${ }^{7}$ that the properties of the Lenard-Balescu model can be investigated more effectively if the electrons are logically divided into two different subspecies, according to their starting velocities, as the evolution of the two subdistributions provides considerable insight into the properties of the LenardBalescu collision operator.

The plasma model presented above can be described by different kinetic theories, with different levels of accuracy; the difference among them consists in the representation of

${ }^{\text {a)}}$ Electronic mail: lapenta@lanl.gov the collision operator, which can be expressed as an expansion in the small plasma parameter $1 /\left(n \lambda_{D}\right),{ }^{8}$ the inverse of the number of plasma particles in one Debye length.

At the zeroth order, the Vlasov model is obtained. It predicts that both the total distribution and the two subdistributions conserve their shape during the evolution.

Retaining terms proportional to $1 /\left(n \lambda_{D}\right)$ in the expansion, the Lenard-Balescu equation is obtained. It shows that, while the total distribution maintains its shape, the two subspecies mix and this mixing takes place in a time scale proportional to $1 /\left(n \lambda_{D}\right)$.

From the exact kinetic theory, the thermalization of the total distribution is also expected. ${ }^{9}$ This is not predicted by the Lenard-Balescu theory; indeed thermalization is a process at least proportional to $1 /\left(n \lambda_{D}\right)^{2}$.

The present work provides an accurate numerical investigation of the Lenard-Balescu equation remarking also the differences among the three models described above and providing a numerical confirmation of all the theoretical predictions. To achieve this end we will use two computer codes.

First, we solve the Lenard-Balescu equations using a simple numerical method. We have studied various implicit and explicit methods and have validated their accuracy. The results of this code is the numerical solution of the LenardBalescu model which, of course, retains the same approximations typical of the Lenard-Balescu approach. Note that the Vlasov model can also be simulated by this same code, simply removing the contribution of the collision integral.

Second, we have developed a simulation code that solves numerically the exact kinetic model with high accuracy, providing the exact plasma response. This approach can be considered as a computer experiment that represents exactly an idealized real experiment conducted with real plasma particles. No approximations are made. In fact, in agreement with the kinetic theories, the exact simulation of the plasma shows a mixing of the two subspecies with a time 

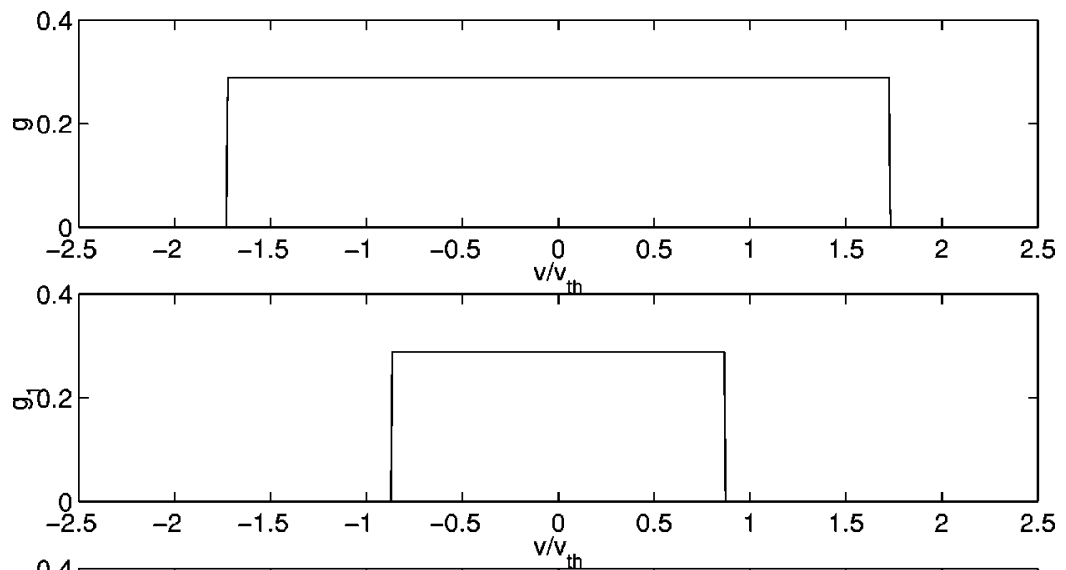

FIG. 1. The averall electron distribution, $g$ $=\left(f m v_{\mathrm{th}}\right) / n$, and the two subdistributions, $g_{1}$ $=\left(f_{1} m v_{\text {th }}\right) / n$ and $g_{2}=\left(f_{2} m v_{\text {th }}\right) / n$, at the starting time.

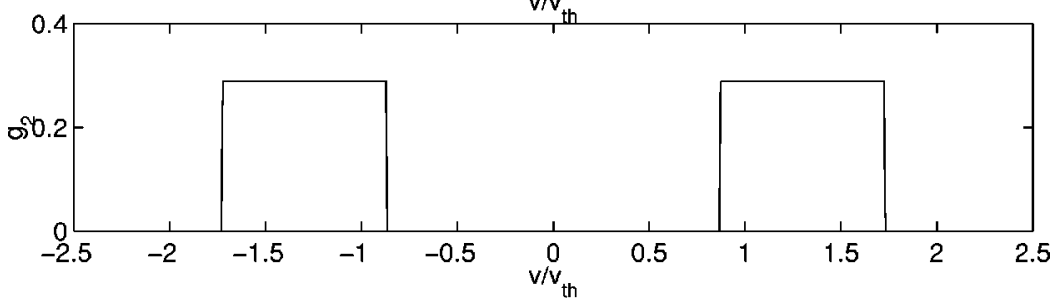

scale proportional to $1 /\left(n \lambda_{D}\right)$ and the thermalization of the particles proportional to $1 /\left(n \lambda_{D}\right)^{2}$.

The two computer codes implemented can be used to experiment directly the properties of the various kinetic models described above (Vlasov, Lenard-Balescu and exact) and they provide new insight for the property of the LenardBalescu collision operator.

First, we can evaluate quantitatively the mixing process described above. The present work is, to our knowledge, the first instance where the evolution governed by the LenardBalescu equation is found by its numerical solution. Also, the mixing of the two subspecies shown by the microscopic simulations can be used as a test for the numerical integration of the Lenard-Balescu equation. Previous studies were limited to evaluate the compatibility of the general trend of the various methods, without actually solving the LenardBalescu equation. In particular, it is shown that the results of the two models agree remarkably well.

Second, the present work provides the derivation and investigation of numerical methods to solve the LenardBalescu equation. A survey of the available literature reveals that essentially no numerical methods have been investigated to approach such models. The present work provides a relatively simple but effective method to solve numerically the Lenard-Balescu equations.

The Lenard-Balescu collision operator is important in many different areas of plasma physics, ranging from the kinetic theory of dusty plasmas ${ }^{10}$ to the particle beamsplasma interactions and laser-plasma interactions. ${ }^{11-13}$ Thus, the present work constitutes the first step towards the numerical solution of the Lenard-Balescu equation in more complex physical situations where the numerical solutions presented here can be used as a benchmark for more comprehensive codes. We remark, in particular, that the numerical method described in this paper has been successfully applied to solve the kinetic equations of dusty plasma, in order to study the evolution of the ion and electron distribution functions. ${ }^{14}$ Also, it is possible to use the numerical method presented here to provide new insight to basic problems in plasma physics, such as the study of fluctuations, where onedimensional and multiple water bag plasmas, similar to that one considered in this work, have been investigated recently. ${ }^{15}$

The present paper is organized as follows. After the Introduction, Sec. II describes the plasma considered and the kinetic models employable for its description. Section III describes the numerical technique used to integrate the Lenard-Balescu equation. Finally, Sec. IV reports the results of the numerical integration and compares them with the results of the microscopic simulations.

\section{THE KINETIC DESCRIPTION OF THE PLASMA}

We consider a one-dimensional electrostatic plasma, spatially homogeneous. It consists of a large number of identical electrons embedded in a neutralizing uniform and fixed background which represents motionless ions. The electrons are free to move under the effect of the electric field and, when they come in contact, are allowed to cross each other. In order to study the kinetic evolution of the plasma, the initial distribution function is required. At $t=0$, we consider the "water bag" distribution" for the electrons (see Fig. 1),

$$
\begin{aligned}
f(r, p, t=0)= & \frac{n}{2 \sqrt{3} m v_{\mathrm{th}}}\left[\theta\left(p+\sqrt{3} m v_{\mathrm{th}}\right)\right. \\
& \left.-\theta\left(p-\sqrt{3} m v_{\mathrm{th}}\right)\right],
\end{aligned}
$$

where $m$ is the mass of each electron, $n$ is the electron density, and $v_{\text {th }}$ is the electron thermal velocity.

In order to make the study presented here more insightful, the electrons are artificially divided in two different subdistributions, according to their starting velocities. One subdistribution (shown in Fig. 1) is made by the electrons sampled from the function, 


$$
\begin{aligned}
f_{1}(r, p, t=0)= & \frac{n}{2 \sqrt{3} m v_{\text {th }}}\left[\theta\left(p+\frac{\sqrt{3}}{2} m v_{\text {th }}\right)\right. \\
& \left.-\theta\left(p-\frac{\sqrt{3}}{2} m v_{\text {th }}\right)\right]
\end{aligned}
$$

which corresponds to the center of the "water bag" distribution, while the other subdistribution (also shown in Fig. 1) is made by the electrons in the tails of the distribution,

$$
\begin{aligned}
f_{2}(r, p, t=0)= & \frac{n}{2 \sqrt{3} m v_{\text {th }}}\left[\theta\left(p+\sqrt{3} m v_{\text {th }}\right)\right. \\
& -\theta\left(p+\frac{\sqrt{3}}{2} m v_{\text {th }}\right)+\theta\left(p-\frac{\sqrt{3}}{2} m v_{\text {th }}\right) \\
& \left.-\theta\left(p-\sqrt{3} m v_{\text {th }}\right)\right] .
\end{aligned}
$$

Clearly the two subdistributions are chosen so that

$$
f_{1}(r, p, t=0)+f_{2}(r, p, t=0)=f(r, p, t=0) \text {. }
$$

The evolution of the distribution function $f(r, p, t)$ and of the two subdistributions $f_{1}(r, p, t)$ and $f_{2}(r, p, t)$ can be described using different kinetic models. In particular, the description of the Lenard-Balescu kinetic model is more accurate than that of the Vlasov model. There is a reason for this, and a brief review of some aspects of the plasma kinetic theory can elucidate this point.

In a one-dimensional electrostatic plasma, for the species (or the subspecies) $\alpha$ with particle charge $e_{\alpha}$, the well known general Boltzmann equation states that

$$
\begin{aligned}
& \frac{\partial f_{\alpha}(r, p, t)}{\partial t}+\frac{p}{m} \frac{\partial f_{\alpha}(r, p, t)}{\partial r}+e_{\alpha} E(r, t) \frac{\partial f_{\alpha}(r, p, t)}{\partial p} \\
& =I_{\alpha}(r, p, t) .
\end{aligned}
$$

The difference between various kinetic theories consists in the representation of the collision operator $I_{\alpha}(r, p, t)$. The collision operator can be expanded using the small plasma parameter $1 /\left(n \lambda_{D}\right)$, the inverse of the number of particles in a Debye length. This parameter is related to the importance of the collisions in the plasma evolution: larger values of this parameter correspond to conditions where collisions are more important.

At the zeroth order of the expansion, the Vlasov model is obtained: all terms proportional to $1 /\left(n \lambda_{D}\right)$ and those of higher order are neglected. At the first order, the LenardBalescu equation is deduced by retaining terms proportional to $1 /\left(n \lambda_{D}\right)$ in the expansion of the collision operator and neglecting those of higher order.

For the plasma described above, the Vlasov theory predicts that both the total distribution, $f$ [Eq. (1)], and the two subdistributions, $f_{1}$ and $f_{2}$ [Eqs. (2)-(3)], conserve their shape during the evolution. In fact, the Vlasov theory states that

$$
I_{\alpha}(r, p, t)=0
$$

always, because the Vlasov model does not take into consideration any discrete particle effect. Therefore, according to the Vlasov model, if there is no macroscopic electric field and if the distribution functions are spatially uniform (as in the case considered here), the total distribution function and the two subdistributions do not evolve,

$$
\frac{\partial f(r, p, t)}{\partial t}=0, \quad \frac{\partial f_{1}(r, p, t)}{\partial t}=0, \quad \frac{\partial f_{2}(r, p, t)}{\partial t}=0 .
$$

Accordingly, the Vlasov equation is not useful to describe the evolution of the plasma considered here and the attention will be focused on the Lenard-Balescu model.

The Lenard-Balescu equation shows that, while the total distribution maintains its shape, the two subdistributions mix. In a one-dimensional plasma, the general form of the Lenard-Balescu collision operator is ${ }^{3,4}$

$$
\begin{aligned}
I_{\alpha}(p)= & \frac{1}{2} \sum_{\beta} e_{\alpha}^{2} e_{\beta}^{2} \frac{\partial}{\partial p} \iint \frac{1}{k^{2}|\epsilon(k, k v)|^{2}} \cdot \delta\left(k\left(v-v^{\prime}\right)\right) \\
& \times\left[f_{\beta}\left(p^{\prime}\right) \frac{\partial f_{\alpha}(p)}{\partial p}-f_{\alpha}(p) \frac{\partial f_{\beta}\left(p^{\prime}\right)}{\partial p^{\prime}}\right] d p^{\prime} d k,
\end{aligned}
$$

where the sum must be extended to all the species (or subdistributions) present in the plasma and where the dielectric function is

$$
\epsilon(k, k v)=\epsilon_{0}+\sum_{\alpha} \frac{e_{\alpha}^{2}}{k^{2}} \int \frac{\partial f_{\alpha}\left(m_{\alpha} v^{\prime}\right)}{\partial v^{\prime}} \frac{d v^{\prime}}{v-v^{\prime}} .
$$

The integration over $d p^{\prime}$ in Eq. (8) can be performed analytically and the Lenard-Balescu collision operator takes the more insightful form,

$$
\begin{aligned}
I_{\alpha}(p)= & \frac{1}{2} \sum_{\beta} e_{\alpha}^{2} e_{\beta}^{2} \frac{m_{\beta}}{m_{\alpha}} \frac{\partial}{\partial v}\left[\left(\int \frac{d k}{|k|^{3}|\epsilon(k, k v)|^{2}}\right)\right. \\
& \left.\cdot\left(f_{\beta}(p) \frac{\partial f_{\alpha}(p)}{\partial p}-f_{\alpha}(p) \frac{\partial f_{\beta}(p)}{\partial p}\right)\right] .
\end{aligned}
$$

Equation (10) shows more clearly that the self-collisions do not give any contribution to the Lenard-Balescu collision operator; in fact, no contribution is given to the sum by the case $\beta=\alpha$.

Regarding the plasma considered here, this means that no evolution is possible for the overall distribution function $f(r, p, t)$ (as, in this case, only self-collisions are present in the collision operator) but, in general, the two subdistributions can change,

$$
\frac{\partial f_{1}(r, p, t)}{\partial t} \neq 0, \quad \frac{\partial f_{2}(r, p, t)}{\partial t} \neq 0
$$

because the collisions between the two subdistributions affect each other. Since the Lenard-Balescu theory takes into consideration terms proportional to $1 /\left(n \lambda_{D}\right)$, the mixing of the two subdistributions takes place in times proportional to $1 /\left(n \lambda_{D}\right)$ and typically on a very slow time scale, compared to others, such as the plasma frequency.

According to the Lenard-Balescu theory, the evolution of the two subdistributions can be investigated solving numerically the following coupled system of nonlinear partial differential equations, 
TABLE I. Expectations from the different kinetic models.

\begin{tabular}{lcc}
\hline \hline \multicolumn{1}{c}{ Model } & Mixing & Thermalization \\
\hline Vlasov & No & No \\
Lenard-Balescu & Yes & No \\
Exact & Yes & Yes \\
\hline
\end{tabular}

$$
\begin{aligned}
\frac{\partial f_{1}(p, t)}{\partial t}= & \frac{1}{2} e^{4} \frac{\partial}{\partial v}\left[\left(\int \frac{d k}{|k|^{3}|\epsilon(k, k v)|^{2}}\right)\right. \\
& \left.\times\left(f_{2}(p, t) \frac{\partial f_{1}(p, t)}{\partial p}-f_{1}(p, t) \frac{\partial f_{2}(p, t)}{\partial p}\right)\right], \\
\frac{\partial f_{2}(p, t)}{\partial t}= & \frac{1}{2} e^{4} \frac{\partial}{\partial v}\left[\left(\int \frac{d k}{|k|^{3}|\epsilon(k, k v)|^{2}}\right)\right. \\
& \left.\times\left(f_{1}(p, t) \frac{\partial f_{2}(p, t)}{\partial p}-f_{2}(p, t) \frac{\partial f_{1}(p, t)}{\partial p}\right)\right],
\end{aligned}
$$

where $e(e>0)$ denotes the particle charge.

Below, this kinetic model is studied numerically and the numerical results are compared with the exact model of the plasma.

Finally, we note that, with an exact collision operator, the complete thermalization of the total distribution has to be expected. As thermalization is not described by the LenardBalescu collision operator, it will take place in time proportional to, at most, $1 /\left(n \lambda_{D}\right)^{2}$.

The expectations of the different kinetic descriptions are summarized in Table I.

\section{NUMERICAL INTEGRATION OF THE LENARD-BALESCU EQUATION}

The integration of the Lenard-Balescu equation requires the evaluation of the kinetic parameter,

$$
D(v, t)=\int \frac{d k}{|k|^{3}|\epsilon(k, k v)|^{2}}
$$

present in system (12). The peculiar plasma we are considering let us evaluate analytically this kinetic parameter. In fact, the square modulus of the dielectric constant is

$$
\begin{aligned}
|\epsilon(k, k v)|^{2}= & \left(\epsilon_{0}+\sum_{\alpha} \frac{e_{\alpha}^{2}}{k^{2}} \mathcal{P} \int \frac{\partial f_{\alpha}\left(m_{\alpha} v^{\prime}, t\right)}{\partial v^{\prime}} \frac{d v^{\prime}}{v-v^{\prime}}\right)^{2} \\
& +\left(\left.\pi \sum_{\alpha} \frac{e_{\alpha}^{2}}{k^{2}} \frac{\partial f_{\alpha}\left(m_{\alpha} v^{\prime}, t\right)}{\partial v^{\prime}}\right|_{v^{\prime}=v}\right)^{2}
\end{aligned}
$$

so that the kinetic parameter $D(v, t)$ can be written as

$$
D(v, t)=\int \frac{|k| d k}{\left|\epsilon_{0} k^{2}+A(v, t)\right|^{2}+|B(v, t)|^{2}},
$$

where

$$
A(v, t)=\sum_{\alpha} e_{\alpha}^{2} \mathcal{P} \int \frac{\partial f_{\alpha}\left(m_{\alpha} v^{\prime}, t\right)}{\partial v^{\prime}} \frac{d v^{\prime}}{v-v^{\prime}},
$$

$$
B(v, t)=\left.\pi \sum_{\alpha} e_{\alpha}^{2} \frac{\partial f_{\alpha}\left(m_{\alpha} v^{\prime}, t\right)}{\partial v^{\prime}}\right|_{v^{\prime}=v}
$$

and the integration over $d k$ can be performed analytically leading to

$$
D(v, t)= \begin{cases}\frac{1}{\epsilon_{0}|B(v, t)|} \operatorname{arccot}\left(\frac{A(v, t)}{|B(v, t)|}\right) \quad \text { if } B(v, t) \neq 0 \\ \frac{1}{\epsilon_{0} A(v, t)} \quad \text { if } B(v, t)=0 .\end{cases}
$$

For the peculiar plasma we are considering, the evaluation of the kinetic parameter $A(v, t)$ and $B(v, t)$ can be performed analytically. The results are that

$$
A(v, t)=A(v)=\frac{n e^{2}}{2 \sqrt{3} m v_{\mathrm{th}}}\left(\frac{1}{v+\sqrt{3} m v_{\mathrm{th}}}-\frac{1}{v-\sqrt{3} v_{\mathrm{th}}}\right),
$$

$$
\begin{aligned}
B(v, t)=B(v)= & \pi \frac{n e^{2}}{2 \sqrt{3} m v_{\text {th }}}\left[\delta\left(v+\sqrt{3} m v_{\text {th }}\right)\right. \\
& \left.-\delta\left(v-\sqrt{3} v_{\text {th }}\right)\right],
\end{aligned}
$$

where we used that the Lenard-Balescu equation does not predict any change for the overall distribution: the consequence is that both $A(v, t)$ and $B(v, t)$ are constant with respect to time. Consequently, it is $D(v, t)=D(v)$ and, remembering Eq. (18) and the value $A(v)$ given by Eq. (19), it is simply

$$
D(v)=m \frac{v^{2}-3 v_{\mathrm{th}}^{2}}{n e^{2} \epsilon_{0}}
$$

when $v \neq \pm \sqrt{3} v_{\text {th }}$.

The next step necessary to solve the Lenard-Balescu equation is the derivation of a numerical scheme. A finitedifference algorithm is chosen. ${ }^{16}$

The domain of integration is discretized using a rectangular grid, with nodes $\left(p_{j}, t_{n}\right)$ and spacing $\Delta p$ and $\Delta t$ and the numerical integration provides the approximated values of the distribution functions evaluated in the nodes of the grid, $f_{\alpha}^{j, n}$. The explicit Euler algorithm for the numerical integration leads to the following discretized formulation:

$$
\begin{aligned}
\frac{f_{1}^{j, n+1}-f_{1}^{j, n}}{\Delta t}= & \frac{e^{4}}{2} \frac{D^{j+1 / 2}}{\Delta v}\left(\frac{f_{2}^{j+1, n}+f_{2}^{j, n}}{2} \frac{f_{1}^{j+1, n}-f_{1}^{j, n}}{\Delta p}\right. \\
& \left.-\frac{f_{1}^{j+1, n}+f_{1}^{j, n}}{2} \frac{f_{2}^{j+1, n}-f_{2}^{j, n}}{\Delta p}\right) \\
& -\frac{e^{4}}{2} \frac{D^{j-1 / 2}}{\Delta v}\left(\frac{f_{2}^{j, n}+f_{2}^{j-1, n}}{2} \frac{f_{1}^{j, n}-f_{1}^{j-1, n}}{\Delta p}\right. \\
& \left.-\frac{f_{1}^{j, n}+f_{1}^{j-1, n}}{2} \frac{f_{2}^{j, n}-f_{2}^{j-1, n}}{\Delta p}\right),
\end{aligned}
$$




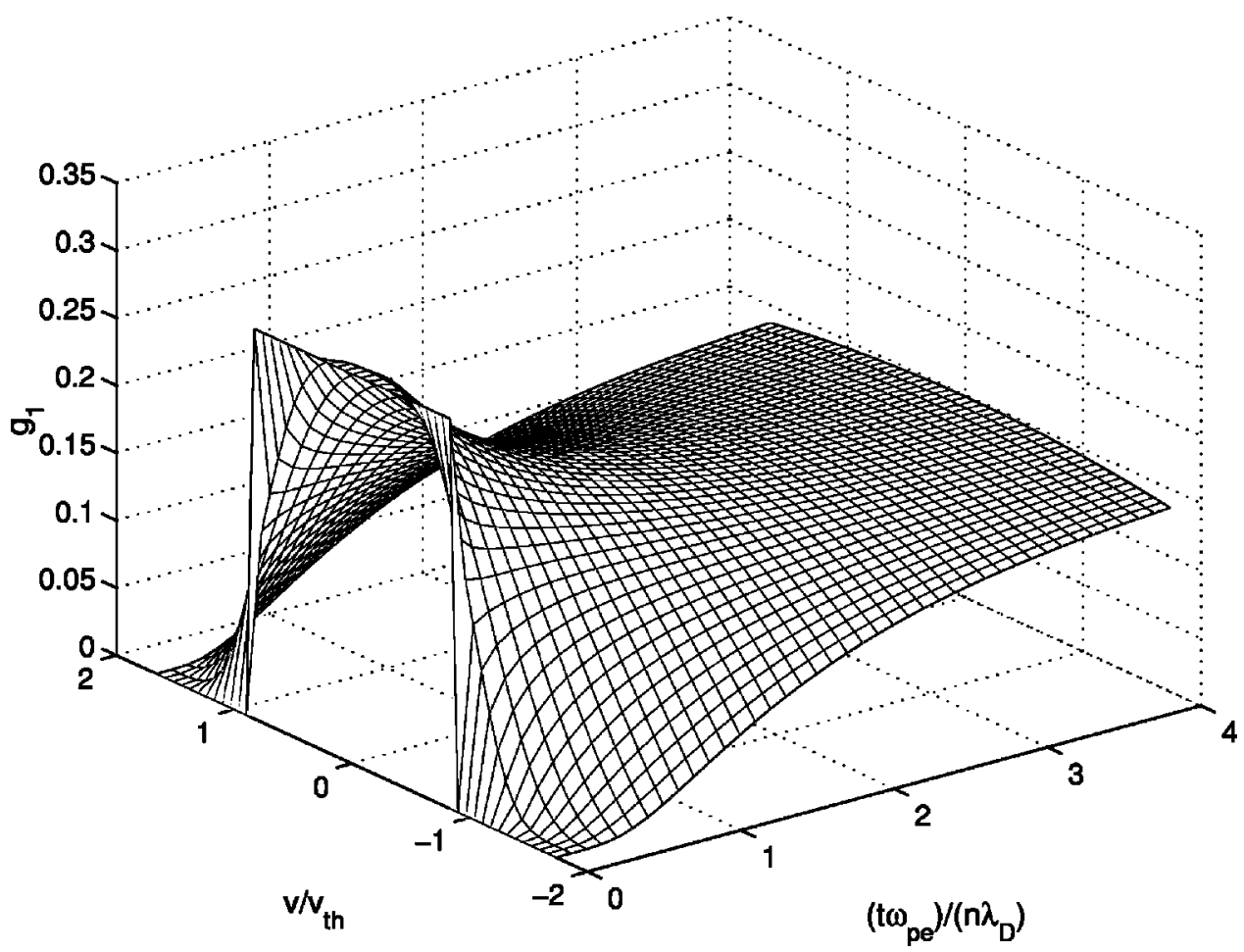

FIG. 2. Distribution function $g_{1}$ $=\left(f_{1} m v_{\text {th }}\right) / n$ obtained solving the Lenard-Balescu equations.

$$
\begin{aligned}
\frac{f_{2}^{j, n+1}-f_{2}^{j, n}}{\Delta t}= & \frac{e^{4}}{2} \frac{D^{j+1 / 2}}{\Delta v}\left(\frac{f_{1}^{j+1, n}+f_{1}^{j, n}}{2} \frac{f_{2}^{j+1, n}-f_{2}^{j, n}}{\Delta p}\right. \\
& \left.-\frac{f_{2}^{j+1, n}+f_{2}^{j, n}}{2} \frac{f_{1}^{j+1, n}-f_{1}^{j, n}}{\Delta p}\right) \\
& -\frac{e^{4}}{2} \frac{D^{j-1 / 2}}{\Delta v}\left(\frac{f_{1}^{j, n}+f_{1}^{j-1, n}}{2} \frac{f_{2}^{j, n}-f_{2}^{j-1, n}}{\Delta p}\right. \\
& \left.-\frac{f_{2}^{j, n}+f_{2}^{j-1, n}}{2} \frac{f_{1}^{j, n}-f_{1}^{j-1, n}}{\Delta p}\right) .
\end{aligned}
$$

Note that the right-hand side is evaluated using the values of the function at time step $n$. It follows that the values of the distribution functions $f_{\alpha}^{j, n+1}$ can be explicitly computed at time $n+1$, when their values at time $n$ are known. In fact, system (22) provides directly the values of the distribution functions at time $n+1$ simply inserting their values at time $n$ in Eqs. (22). The explicit algorithm is conditionally-stable and a small (but still manageable) time step is required to perform a stable integration.

At the starting time, system (12) affirms that, except for $v= \pm \sqrt{3} v_{\text {th }} / 2$, the collision integrals vanish, as the two subspecies $f_{1}$ and $f_{2}$ do not overleap. However, for $v= \pm \sqrt{3} v_{\text {th }} / 2$, the collision integrals do not vanish and present a singularity due to the derivatives of the two subdistributions. In the numerical scheme (22), because of the discretization, the two subdistributions overleap in an interval $\Delta p$ wide and the singularities are smoothed: the analytical behavior is found in the limit $\Delta p \rightarrow 0$. Indeed, the singularities contained in the analytical expressions are obtained rigorously in this limit process. Reducing the size of the interval $\Delta p$, the numerical sequence of results obtained tends asymptotically to the exact evolution of the two sub- distribution functions: in particular it is found that the two subdistributions, at time $t=0$, show a finite evolution, despite the fact that they only overlap in the two points $v= \pm \sqrt{3} v_{\mathrm{th}} / 2$.

We remark that the explicit algorithm satisfies the following important conservation property,

$$
f_{1}^{j, n}+f_{2}^{j, n}=f_{1}^{j, 0}+f_{2}^{j, 0}
$$

for every $n$, as prescribed by the analytic Lenard-Balescu equation.

This property is still true if an implicit algorithm is implemented, as would be the case if the right-hand side of system (22) is evaluated using the distribution functions at time $n+1$. Nevertheless, the implicit algorithm leads to a very complex system of nonlinear equations, whose solution is rather challenging. An easier alternative would be to evaluate the temporal derivative using the distribution functions partly at time $n$ and partly at time $n+1$. Indeed, if the time derivative for $f_{1}$ is evaluated using the values $f_{1}^{j, n+1}$ and $f_{2}^{j, n}$, and the opposite is done for $f_{2}$, the numerical algorithm implemented leads to a simple tridiagonal system of linear equations. However, property (22) would not be verified, leading to an unconditionally unstable scheme of no practical use.

\section{RESULTS AND COMPARISON WITH THE MICROSCOPIC MODEL}

The numerical scheme (22) has been implemented in a code. The results of the numerical integrations are presented in Figs. 2 and 3, where the shapes of the two subdistribution functions are shown at different time steps. First of all, it is clear that the two subdistributions change, while the overall distribution maintains its aspect; in particular, the two sub- 


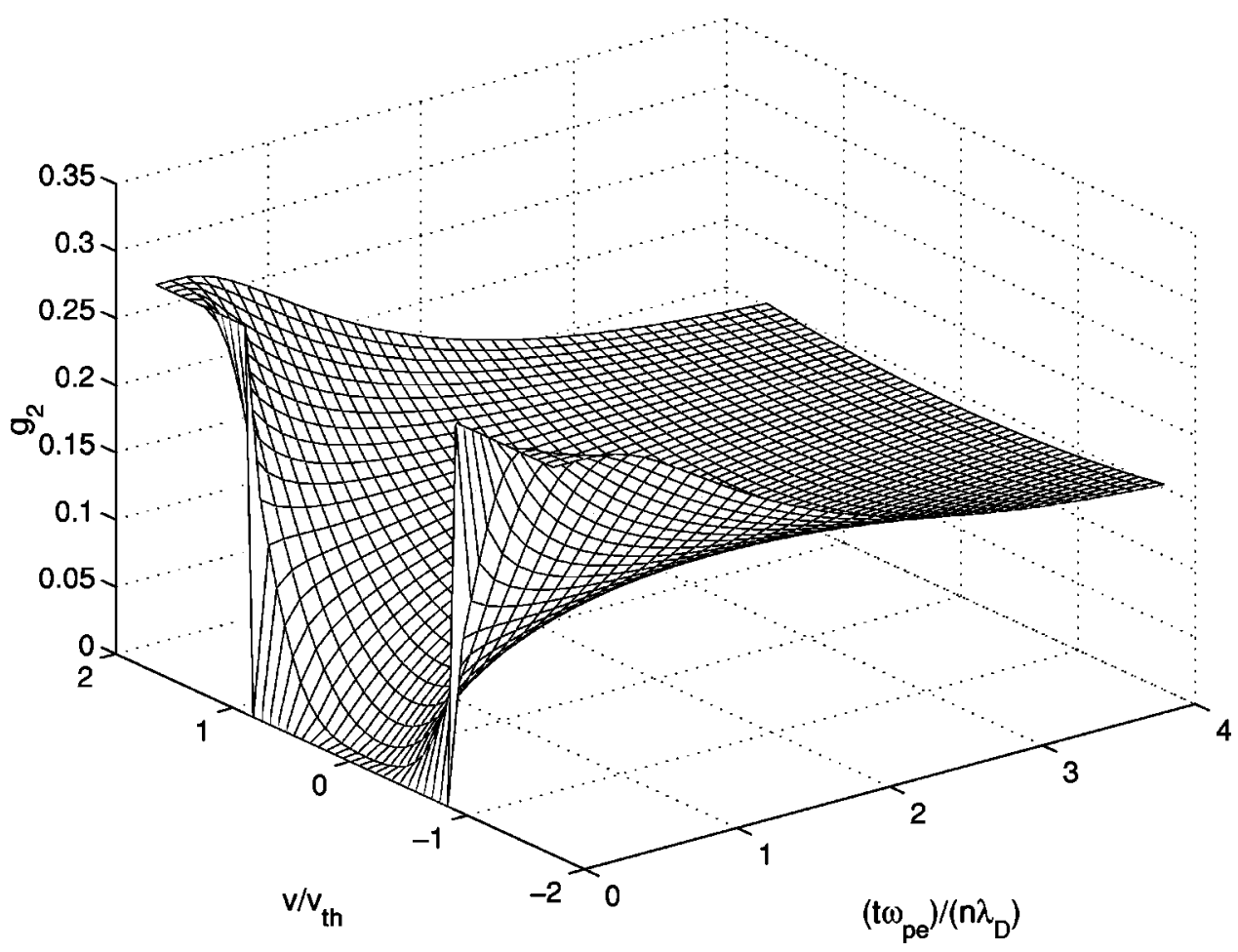

FIG. 3. Distribution function $g_{2}$ $=\left(f_{2} m v_{\text {th }}\right) / n$ obtained solving the Lenard-Balescu equations. distributions lose their initial shape and tend towards the same water bag distribution which has the same width but half height of the total distribution.

The relatively simple plasma considered here enables us to check the precision of the Lenard-Balescu equation and, obviously, the precision of the numerical scheme adopted for its solution as it is possible to compare the results of the numerical integration with the results of an exact microscopic code.

The microscopic model used to perform the simulation is similar to the one described in Refs. 7 and 17 and is described in the Appendix. Here, we shortly remark that the microscopic evolution of the peculiar plasma considered here can be computed exactly; in fact, it is possible to integrate exactly (except for unavoidable round-off errors) the Newton equations for the motion of all the electrons together with the self-consistent Poisson equation. Consequently, from the motion of all the electrons, it is possible to evaluate the general behavior of the plasma. The exact simulation confirms the range of validity of each kinetic theory, showing that the mixing of the two subdistributions occurs on a time scale proportional to $1 /\left(n \lambda_{D}\right)$ and revealing that thermalization is a process of longer time scale occurring in a time proportional to $1 /\left(n \lambda_{D}\right)^{2}$.

To compare the evolution of the plasma provided by the two models, it must be remembered that only phenomena occurring on time scales with order of $1 /\left(n \lambda_{D}\right)$ can be compared. The fact is that phenomena of longer time scale, even if revealed by the exact simulations, transcend the LenardBalescu equation. This means that only the mixing of the two subdistributions, which appears at the beginning of the exact simulations, can be used for the comparison. The comparison between the numerical solution of the Lenard-Balescu equa- tion and the microscopic simulations is shown in Figs. 4 and 5.

In Fig. 4 the two subdistribution functions are plotted at different times together with the histograms representing the velocity distributions reported by the exact simulations. The distribution functions described by the Lenard-Balescu equation and those described by the microscopic simulation are very similar.

The exact simulation and the numerical integration of the Lenard-Balescu equation can also be compared observing the kinetic energies of the electrons belonging to the two subdistributions. Different microscopic simulations, with different values of the plasma parameter $n \lambda_{D}$, have been performed. In the case of the microscopic simulations, the kinetic energies of each subdistributions is evaluated averaging the kinetic energy of the electrons; in the case of the numerical solution of the Lenard-Balescu equation, the kinetic energy is found by discretizing the following integral:

$$
E_{\mathrm{kin}, \alpha}(t)=\frac{1}{2} \int m v^{2} f_{\alpha}(p, t) d p .
$$

Figure 5 shows such kinetic energies, in a dimensionless form. The kinetic energies are plotted as a function of time rescaled by the factor $1 /\left(n \lambda_{D}\right)$; in fact, the Lenard-Balescu collision operator is proportional to $1 /\left(n \lambda_{D}\right)$, and causes an evolution on that rescaled time, as expected. Therefore, Fig. 5 shows that the mixing of the two subdistributions occurs in a time scale proportional to $1 /\left(n \lambda_{D}\right)$ and it is completed approximately when $t \omega_{p e}=4\left(n \lambda_{D}\right)$. The most important result shown by Fig. 5 is that the mixing described by the Lenard-Balescu equation and the mixing described by the microscopic simulation agree remarkably well. 

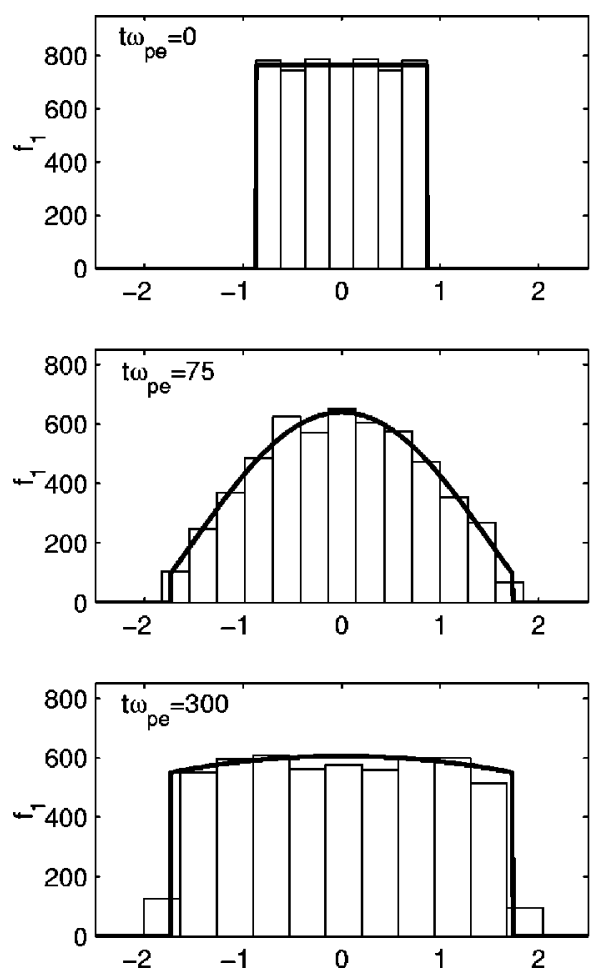
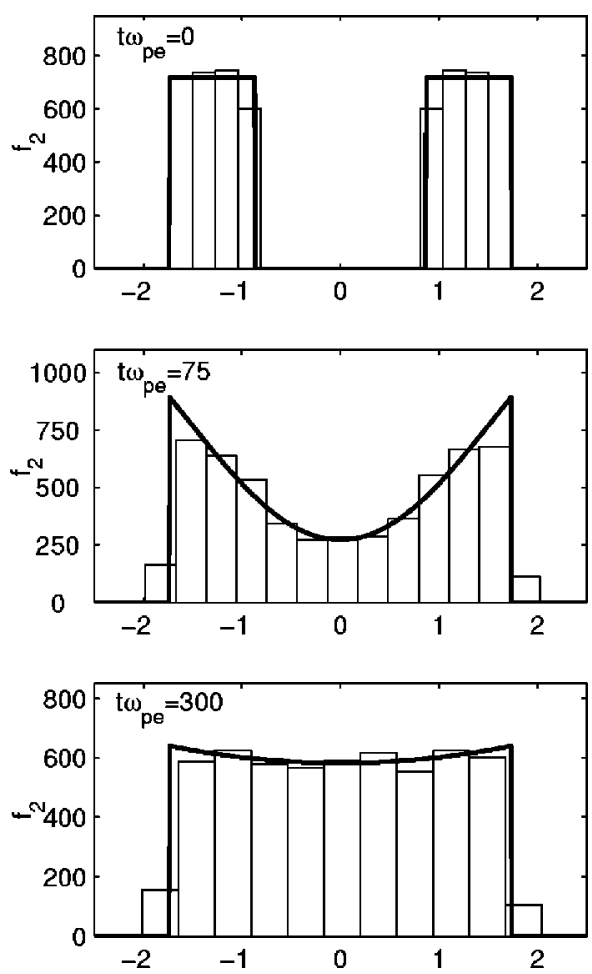

FIG. 4. Histograms rapresenting the number, $N_{p}$, of electrons belonging to the distribution $f_{1}$ and $f_{2}$ according to their velocities at time $t \omega_{p e}=0$ (initial condition), $t \omega_{p e}=75$, and $t \omega_{p e}=300$ and comparison with the distributions found form the numerical solution of the Lenard-Balescu equation. The simulation cosiders $1.1 \cdot 10^{4}$ charges with $n \lambda_{D}=80$.
From Fig. 4, it can be seen that at the end of the simulation, the edges of the subdistribution are not as sharp as they are initially. This is an indicator that, if the simulation is continued, thermalization occurs. However, this phenomenon is of second order in comparison to the mixing of the two subdistributions. In fact, on a longer time scale, the exact simulation shows that the total distribution changes and that the "water bag" distribution tends to become Maxwellian, as shown by Fig. 6.

The thermalization of the total distribution can be measured quantitatively computing the entrophy,

$$
S(t)=-\iint f(r, p, t) \log f(r, p, t) d r d p .
$$

Integral (25) is evaluated numerically dividing the phase space in a number of regions, counting the electrons in each region and discretizing the integral as a sum over all the regions. ${ }^{18}$ Note that $S_{d}$ would remain rigorously constant both for the Vlasov and the Lenard-Balescu method, since $f(r, p, t)=$ const. Consequently, any change of $S_{d}$ is due to processes neglected by the kinetic models considered here.

Figure 7 shows the value of $S_{d}$ as a function of time

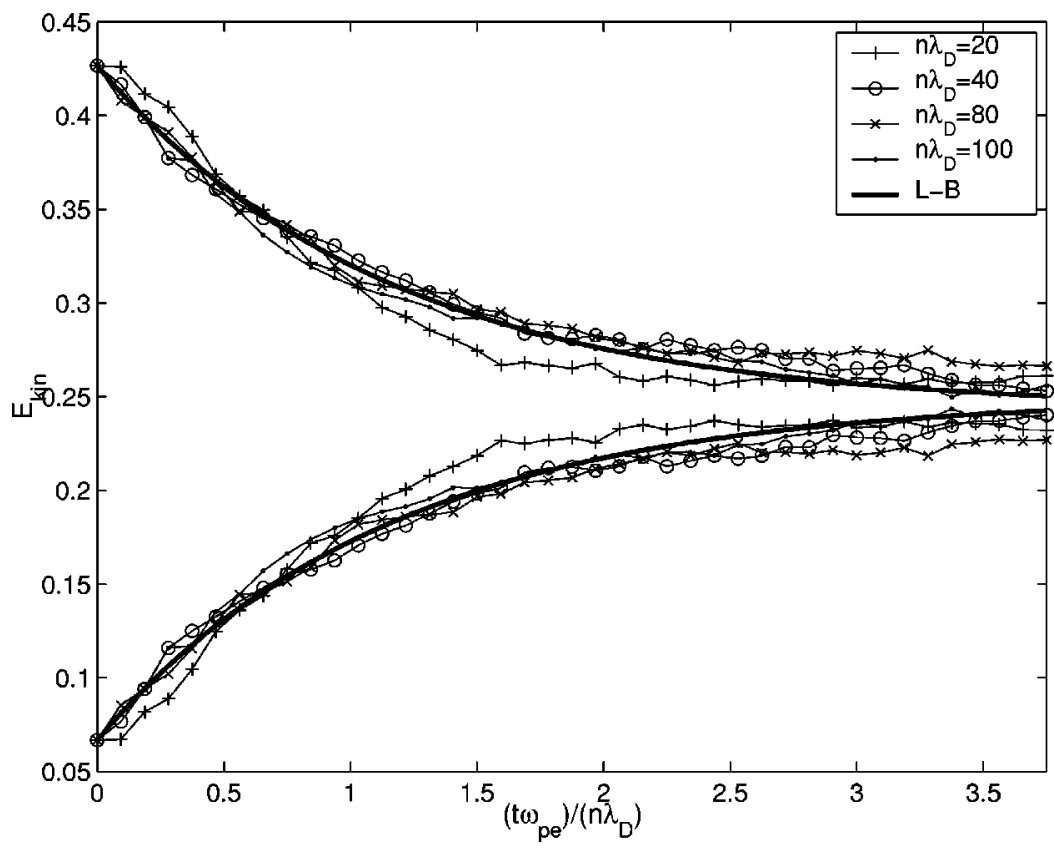

FIG. 5. Evolution of the kinetic energies, $E_{\mathrm{kin}}$, of the two subdistributions, as calculated by the exact simulation, for different values $n \lambda_{D}$, and by the numerical solution of the Lenard-Balescu equation. $E_{\text {kin }}$ is normalized to $m v_{\mathrm{th}}^{2}$. The evolution is followed on the rescaled time $\left(t \omega_{p e}\right) /\left(n \lambda_{D}\right)$. 

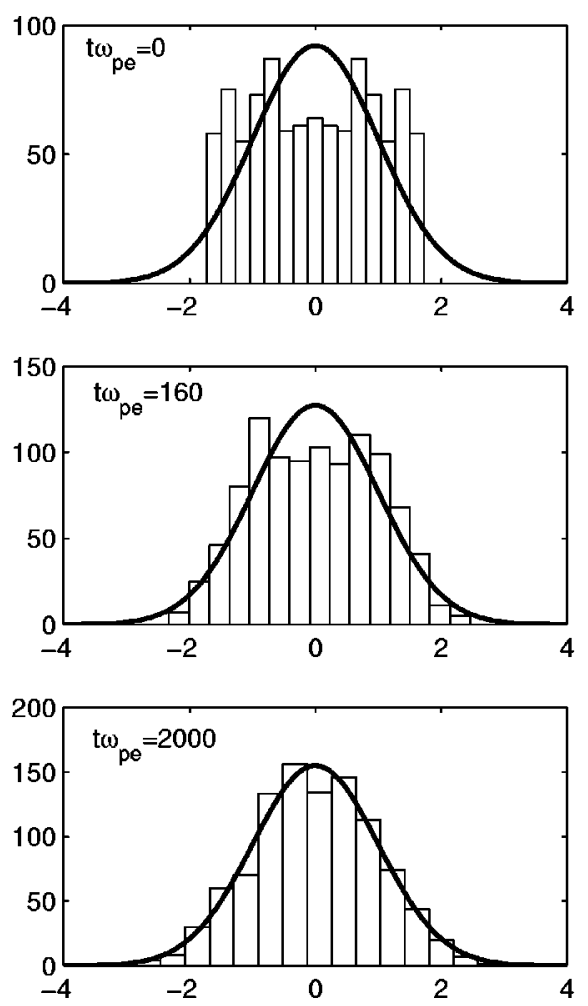

[rescaled by $1 /\left(n \lambda_{D}\right)^{2}$, the time scale at which the thermalization is expected]. The growth of $S_{d}$ in different simulations is similar (except for statistical fluctuations), showing that thermalization develops on a longer time scale than the mixing of the two subdistributions, as thermalization is completed in a time $t \omega_{p e}$ proportional to $\left(n \lambda_{D}\right)^{2}$, confirming the results by Dawson ${ }^{5,6}$ and by Rouet and Feix. ${ }^{7}$

\section{CONCLUSIONS}

In the present work, the kinetics of a relatively simple one-dimensional plasma has been studied. In particular the attention has been focused on the Lenard-Balescu approach.
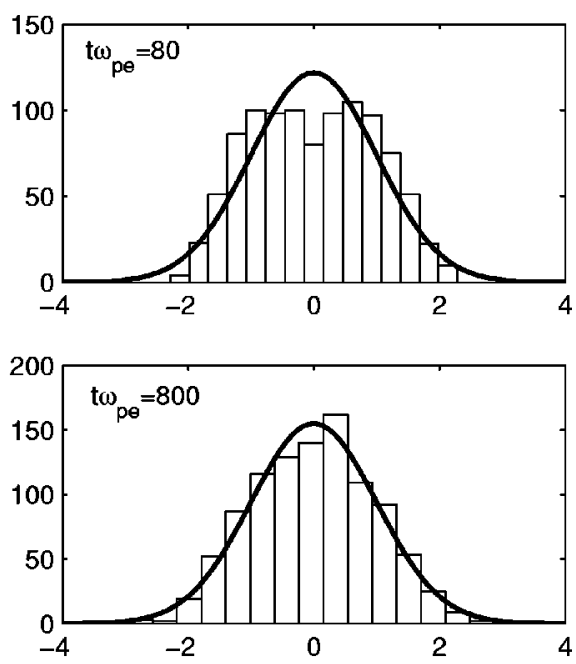

FIG. 6. Histograms representing the number of electrons according to their velocities (a Maxwellian distribution is shown for comparison), at time $t \omega_{p e}=0$ (initial condition), $t \omega_{p e}=80$, $t \omega_{p e}=160, t \omega_{p e}=800, t \omega_{p e}=2000$, and $t \omega_{p e}=4000$. The simulation considers 1000 charges with $n \lambda_{D}=10$.

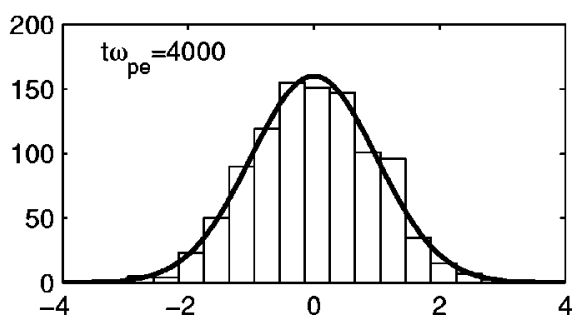

We have developed two computer codes. One solves numerically the Lenard-Balescu equation, the other simulates the exact kinetic model with high accuracy. The two codes have been compared with known results and have been compared with one another.

The study has confirmed the theoretical predictions. Our approach has also provided a quantitative comparison of the various approaches.

The exact simulation has confirmed the range of validity of each kinetic theory and, in particular, the numerical solution of the Lenard-Balescu kinetic equation has shown a remarkable agreement with the exact code in predicting phe-

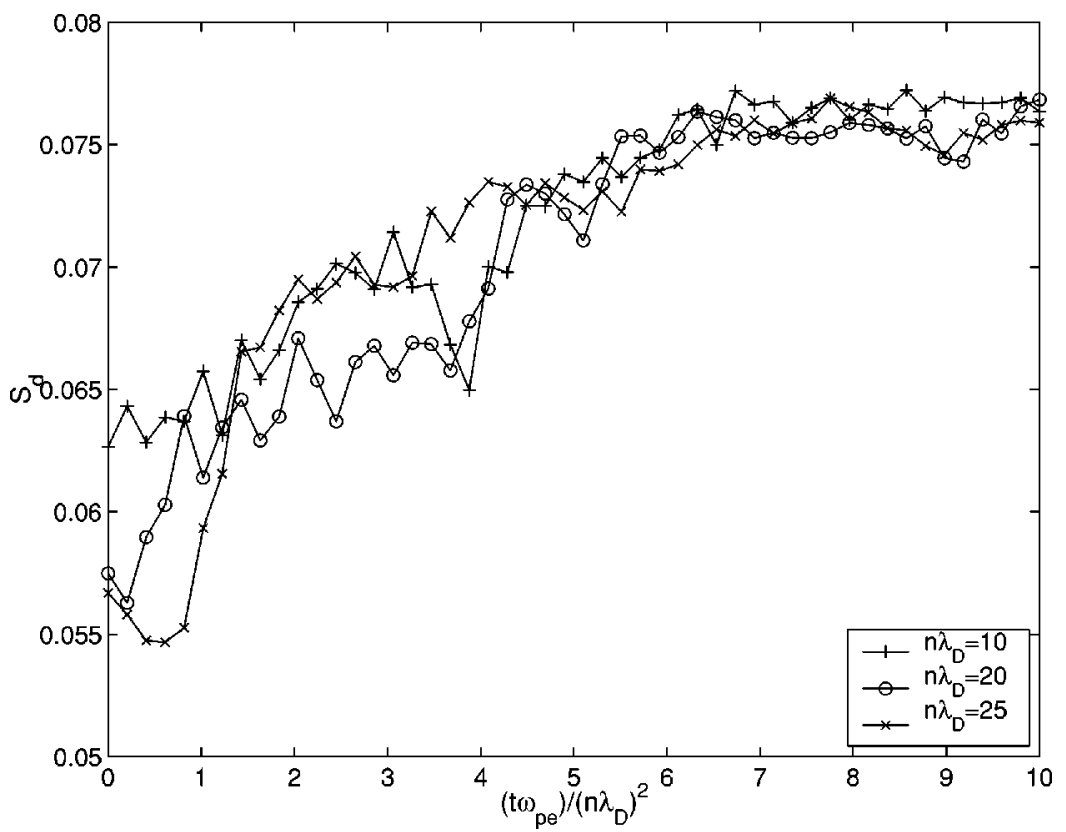

FIG. 7. Growths of the discretized entropy, $S_{d}$, obtained with the exact simulations, for different values $n \lambda_{D}$. The evolution is followed on the rescaled time $\left(t \omega_{p e}\right) /\left(n \lambda_{D}\right)^{2}$. 
nomena which occur on the time scale $1 /\left(n \lambda_{D}\right)$. However, phenomena with longer time scale, which are correctly described by the exact simulations, cannot be reproduced by the Lenard-Balescu equation.

The present work has dealt with the numerical solution of the Lenard-Balescu equation, a topic unknown in previous literature. The results of the simulations presented can also be used as a benchmark for more complex future codes which will face the numerical solution of the LenardBalescu equation in different fields of plasma physics.

\section{ACKNOWLEDGMENTS}

The authors are very grateful to Gianni Coppa and Umberto de Angelis for helpful discussions on the topics reported here.

This research is supported by the United States Department of Energy, under Contract No. W-7405-ENG-36, by NASA, under the "Sun Earth Connection Theory Program," and by the European Community under Contract No. HPRNCT2000-00140.

\section{APPENDIX: MICROSCOPIC MODEL}

This appendix describes the microscopic model used to test the numerical integration of the Lenard-Balescu equation. This model is able to provide the exact motion of all the electrons, as stated by their Newton equation, taking into account self-consistently the Gauss equation governing the electric field.

The Newton law for the $i$ th electron can be written as

$$
m \frac{d^{2} r_{i}(t)}{d t^{2}}=-e E_{i}(t),
$$

where $r_{i}(t)$ is the position of the $i$ th electron at time $t,-e$ denotes the charge of each electron $(e>0)$, and $E_{i}(t)$ is the electric field which acts on the $i$ th electron at time $t$.

The self-consistent Gauss equation states that

$$
\frac{\partial E(r, t)}{\partial r}=-\frac{1}{\epsilon_{0}}\left[\left(\sum_{i} e \delta\left(r-r_{i}(t)\right)\right)-n e\right]
$$

as the charge density present in the physical system is composed by the electrons, with charge $-e \delta\left(r-r_{i}(t)\right)$ and by a uniform positive neutralizing background, with charge density $n e$. The connection between $E_{i}(t)$ and $E(r, t)$ is given by

$$
E_{i}(t)=\frac{1}{2} \lim _{\varepsilon \rightarrow 0}\left[E\left(r_{i}(t)+\varepsilon, t\right)+E\left(r_{i}(t)-\varepsilon, t\right)\right]
$$

in order to take into account possible discontinuities of the electric field. The set of coupled Eqs. (A1)-(A3) summarizes the exact microscopic model of the plasma considered in this study and, by its solution, it is possible to find the exact motion of all the electrons.

The first step towards the complete solution is the integration of the Gauss Law,

$$
E(r, t)=-\frac{e}{\epsilon_{0}}\left\{\sum_{i} \theta\left[r-r_{i}(t)\right]-n r+K(t)\right\},
$$

where $K(t)$ is the value of the integration constant, in principle dependent parametrically upon time, to be determined imposing the boundary conditions. We choose periodic boundary conditions in order to best represent an infinite uniform system; therefore, the electrostatic potential $\varphi(r, t)$ must satisfy the following relationship:

$$
\varphi(0, t)=\varphi(L, t)
$$

at every time, indicating with $L$ the length of the simulated system. This constraint leads to

$$
\int_{0}^{L} E(r, t) d r=0
$$

which determines the value of $K(t)$. After straightforward algebra, it is found that

$$
K(t)=\frac{1}{L} \sum_{i} r_{i}(t)-\frac{1}{2} n L
$$

and, consequently, the value of $K(t)$ depends upon the positions of all the electrons. At the starting time, the initial location of the electrons in the phase space must reflect the distribution function (1); this means that the velocities of the electrons are chosen as a random sample of the water bag distribution (1) and the initial spatial positions are chosen as equally spaced, imposing

$$
r_{i}(t=0)=\frac{2 i-1}{2 n}, \quad i=1,2, \ldots, N,
$$

where $N=n L$ is the total number of particles followed during the simulation. In this way, it is

$$
\begin{aligned}
K(t=0) & =\frac{1}{L} \sum_{i} r_{i}(t=0)-\frac{1}{2} n L \\
& =\frac{1}{L} \sum_{i} \frac{2 i-1}{2 n}-\frac{1}{2} n L=0
\end{aligned}
$$

and, as a consequence, the electric field has a sawtooth shape, with $E(r=0, t=0)=0$. At subsequent times, the value of $K(t)$ depends upon the motion of all the electrons, which must be studied carefully.

We remark that a time interval exists in which no collision takes place. In that lapse, the ordering of the electrons along the axis remains constant and, using Eq. (A3), Eq. (A1) becomes

$$
m \frac{d^{2} x_{i}(t)}{d t^{2}}=-\frac{e^{2} n}{\epsilon_{0}} x_{i}(t)+\frac{e^{2}}{\epsilon_{0}} K(t),
$$

where expression (A7) for $K(t)$ can be inserted and where $x_{i}(t)$ is defined as the distance of the $i$ th electron from its initial position,

$$
x_{i}(t)=r_{i}(t)-r_{i}(t=0) .
$$

Writing the Newton equations for all the electrons and summing them, it is possible to obtain the following system of differential equations,

$$
\frac{d v_{\mathrm{CM}}(t)}{d t}=0
$$




$$
\frac{d x_{\mathrm{CM}}(t)}{d t}=v_{\mathrm{CM}}(t)
$$

which describes the position, $x_{\mathrm{CM}}$, and the velocity, $v_{\mathrm{CM}}$, of the center-of-mass, defined as

$$
x_{\mathrm{CM}}(t)=\sum_{i} \frac{x_{i}(t)}{N}, \quad v_{\mathrm{CM}}(t)=\sum_{i} \frac{v_{i}(t)}{N}=\frac{d}{d t}\left(x_{\mathrm{CM}}\right) .
$$

Thanks to the initial conditions, it is

$$
\begin{aligned}
& v_{\mathrm{CM}}(t)=0, \\
& x_{\mathrm{CM}}(t)=0,
\end{aligned}
$$

and, until no collisions occur, it implies

$$
K(t)=0 .
$$

Consequently, from Eq. (A10) and Eq. (A15), it is possible to conclude that

$$
\frac{d^{2} x_{i}(t)}{d t^{2}}=-\omega_{p e}^{2} x_{i}(t)
$$

and, until any collision takes place, the electrons are governed by the equation of the harmonic oscillator with frequency $\omega_{p e}$.

During their motion, the electrons can come in contact, and a collision takes place. It is assumed that elastic collisions occur: from the conservation of momentum and kinetic energy, and letting the particles cross each other, it results that during a collision, the colliding particles simply exchange their order, maintaining their previous velocities. Consequently, the quantities of system (A12) do not have any discontinuity and it is always $K(t)=0$. This means that, after each collision, the colliding electrons will still move as harmonic oscillators, but about new equilibrium points; the colliding particles, in fact, simply exchange their equilibrium points. We remark that a collision between two electrons does not influence the motion of all the other electrons.

This model can be implemented in a code to perform the simulation of the plasma. The only nontrivial aspect of the code is the evaluation of the crossing times between the electrons and the exchange of the equilibrium positions of the electrons involved in the collisions. We use a method presented in Ref. 17.

\footnotetext{
${ }^{1}$ A. Lenard, Ann. Phys. (N.Y.) 3, 390 (1960).

${ }^{2}$ R. Balescu, Phys. Fluids 3, 52 (1960).

${ }^{3}$ Yu.L. Klimontovich, The Statistical Theory of Nonequilibrium Processes in a Plasma (M.I.T. Press, Cambridge, Massachusetts, 1967), pp. 117139.

${ }^{4}$ V.N. Tsytovich, Lectures on Nonlinear Plasma Kinetics (Springer-Verlag, Berlin, 1995).

${ }^{5}$ J. Dawson, Phys. Fluids 5, 445 (1962).

${ }^{6}$ J. Dawson, Phys. Fluids 7, 419 (1964).

${ }^{7}$ J.L. Rouet and M.R. Feix, Phys. Fluids B 3, 1830 (1991).

${ }^{8}$ P. Lotte and M.R. Feix, J. Plasma Phys. 31, 141 (1984).

${ }^{9}$ K. Huang, Statistical Mechanics (Wiley, New York, 1963).

${ }^{10}$ V.N. Tsytovich and U. de Angelis, Phys. Plasmas 6, 1093 (1999).

${ }^{11}$ B. Strege and W.D. Kraeft, Laser Part. Beams 10, 227 (1992).

${ }^{12}$ N. Peyraud-Cuenca and P. Faucher, J. Plasma Phys. 60, 393 (1998).

${ }^{13}$ R. Shneider, Contrib. Plasma Phys. 41, 315 (2001).

${ }^{14}$ P. Ricci, G. Lapenta, U. de Angelis, and V.N. Tsytovich, Phys. Plasmas 8, 769 (2001).

${ }^{15}$ J.L Rouet and M.R. Feix, Phys. Rev. E 59, 73 (1999).

${ }^{16}$ K.W. Morton and D.F. Mayers, Numerical Solution of Partial Differential Equations (Cambridge University Press, Cambridge, 1994).

${ }^{17}$ M.R. Feix, in Nonlinear Effects in Plasmas, edited by G. Kalman and M.R. Feix (Gordon and Breach, New York, 1969), pp. 151-154.

${ }^{18}$ C.J. Reidl, Jr. and B.N. Miller, Astrophys. J. 318, 248 (1962).
} 